\title{
CORRELACIÓN ENTRE LOS ÍNDICES GLOBALES DE LA PERIMETRÍA AUTOMATIZADA CONVENCIONAL Y LOS PARÁMETROS TOPOGRÁFICOS PAPILARES (HEIDELBERG RETINA TOMOGRAPH II)
}

\author{
CORRELATION BETWEEN STANDARD AUTOMATED \\ PERIMETRY GLOBAL INDICES AND HEIDELBERG RETINA \\ TOMOGRAPH PARAMETERS
}

\author{
PÉREZ-ÍÑIGO A ${ }^{1}$, POLO V ${ }^{1}$, LARROSA JM ${ }^{1}$, FERRERAS A ${ }^{1}$, SÁNCHEZ-CANO A ${ }^{2}$, \\ MARTÍNEZ-DE-LA-CASA JM ${ }^{3}$, HONRUBIA FM ${ }^{1}$
}

\section{RESUMEN}

Objetivo: Correlacionar los parámetros estructurales del nervio óptico obtenidos mediante Heidelberg Retina Tomograph II (HRT) con los índices perimétricos de la perimetría automatizada convencional. Metodos: Se incluyeron 101 sujetos normales, 247 hipertensos oculares [presión intraocular (PIO) elevada con perimetría automatizada convencional normal] y 102 sujetos glaucomatosos $(\mathrm{PIO}>21 \mathrm{mmHg}$ y perimetría automatizada convencional alterada). Solo se incluyó un ojo de cada sujeto para el análisis. La perimetría automatizada se realizó mediante un perímetro Humphrey Field Analyzer (umbral completo, 24-2). El estudio papilar se realizó mediante láser confocal de barrido Heidelberg Retina Tomograph (HRT II). Se realizó un estudio de los coeficientes de correlación (Pearson) entre los parámetros topográficos papilares y los índices perimétricos en el total de sujetos y en cada uno de los grupos de estudio, ya que las muestras presentaban una distribución de sus valores ajustada a la normalidad.

\section{ABSTRACT}

Purpose: To correlate the optic nerve head topographic parameters measured by the Heidelberg Retina Tomograph II (HRT) with the perimetric indices of standard automated perimetry (SAP).

Methods: This study included 101 normal subjects, 247 ocular hypertensive eyes (increased intraocular pressure with normal SAP) and 102 glaucomatous subjects (IOP above $21 \mathrm{~mm} \mathrm{Hg}$ and abnormal standard automated perimetry). Only one eye was randomly chosen from each subject for the study. The visual field was evaluated by means of Humphrey Field Analyzer (24-2 full threshold strategy). The HRT II (Heidelberg Engineering) was used to acquire and measure the optic disc topographic parameters. Pearson correlations between topographic data and perimetric indices were performed for the total sample and each group of patients. The distribution of values obtained in the samples was normal.

Results: A significant correlation was found between several optic disc parameters and the global indi-

\footnotetext{
Recibido: 6/6/05. Aceptado: 6/6/07.

1 Doctor en Medicina. Hospital Universitario Miguel Servet. Zaragoza. España.

2 Licenciada en Ciencias Físicas, especialidad óptica. Hospital Universitario Miguel Servet. Zaragoza. España.

3 Doctor en Medicina. Hospital Clínico Universitario. Madrid. España.

Este trabajo ha sido parcialmente financiado por la Red Nacional de Investigación Cooperativa: Prevención de la Ceguera. Subproyecto de Glaucoma.
}

Correspondencia:

M. ${ }^{a}$ Aránzazu Pérez Íñigo

Paseo Sagasta, $15,9 .^{\circ} \mathrm{C}$

50008 Zaragoza

España

E-mail: aperezinigo@ hotmail.com 
Resultados: Se observaron correlaciones significativas entre varios parámetros de la cabeza del nervio óptico y los índices globales de la perimetría automatizada convencional. En el total de sujetos y en el grupo glaucoma, las correlaciones más fuertes con los índices del campo visual se obtuvieron con el área (total: $r=0,32 ; \mathrm{p}=4,14 \times 10^{-11} /$ glaucoma: $\mathrm{r}=0,28$; $\mathrm{p}=0,004)$ y volumen del anillo neurorretiniano (total: $\mathrm{r}=0,26 ; \mathrm{p}=1,55 \times 10^{-7} / \mathrm{glaucoma} \mathrm{r}=0,26$; $\mathrm{p}=0,006$ ), el cociente de área excavación/disco y anillo/disco, el índice de morfología papilar, el área de corte de la capa de fibras nerviosas de la retina y las funciones discriminantes FSM y RB. El grupo de hipertensión ocular obtuvo pocas correlaciones significativas.

Conclusiones: Las correlaciones observadas entre la perimetría automatizada convencional y HRT II, permiten mejorar el conocimiento del daño glaucomatoso y facilitan la toma de decisiones con los resultados de todas las pruebas de las que disponemos en la actualidad.

Palabras clave: Glaucoma, diagnóstico, HRT, nervio óptico, perimetría.

\section{INTRODUCCIÓN}

El glaucoma crónico simple es una neuropatía progresiva caracterizada por la existencia de alteraciones estructurales a nivel del nervio óptico y de la capa de fibras nerviosas de la retina así como pérdidas funcionales a nivel del campo visual (1-5). La exploración del campo visual y la evaluación clínica del nervio óptico han constituido las piedras angulares en el control evolutivo de la enfermedad (6-13).

El Tomógrafo Retiniano Heidelberg — HRT IIes un láser confocal de barrido de última generación que permite obtener imágenes morfométricas del nervio óptico realizando un análisis papilar objetivo y reproducible, minimizando la subjetividad del operador (14-18).

En un estudio comparativo de diversas técnicas de análisis de imagen llevado a cabo por Nakla et al (19), la precisión diagnóstica del HRT y de la evaluación cualitativa de las fotografías estereoscópicas de la papila fue superior a la del OCT y el analizador de la capa de fibras nerviosas GDx. En otros estudios, sin embargo, no encontraron diferencias significativas en el área bajo la curva ROC entre los mejores parámetros de cada instrumento, pero sí ces of SAP. Rim area, rim volume, cup/disc area ratio, rim/disc area ratio, cup shape measurement, RNFL cross-sectional area, and discriminant functions FSM and RB, showed the strongest correlation with the visual field indices in the total and glaucoma groups (RIM AREA: total group: $r=0.32$; $\mathrm{p}=4.14 \times 10^{-11} /$ glaucoma group: $\mathrm{r}=0.28 ; \mathrm{p}=0.004$. RIM VOLUME: total group: $\mathrm{r}=0.26 ; \mathrm{p}=1.55 \times 10^{-}$ 7 / glaucoma group: $r=0.26 ; p=0.006)$. The ocular hypertensive group showed few significant correlations.

Conclusions: The correlations found between standard automated perimetry and HRT defined topographic parameters allow a better understanding of glaucomatous damage and make decision-making easier (Arch Soc Esp Oftalmol 2007; 82: 401-412).

Key words: Glaucoma, diagnosis, HRT, optic nerve head, perimetry. 
Se excluyeron los individuos con historia previa de patología, cirugía o traumatismo oculares, enfermedades sistémicas de repercusión oftálmica, imposibilidad de realizar o valorar cualquiera de las pruebas incluidas en el protocolo exploratorio (estudio perimétrico, HRT,...) o que incumplían algún criterio de inclusión.

Los sujetos incluidos fueron clasificados en tres grupos de estudio: normal o control, hipertensión ocular (HTO) y glaucoma crónico simple.

- Grupo Control ( $\mathrm{n}=101$ ojos). Todos cumplían los criterios de inclusión. En ninguna de las determinaciones la presión intraocular (PIO) debía estar por encima de los $21 \mathrm{~mm} \mathrm{Hg}$.

- Grupo HTO ( $\mathrm{n}=247$ ojos). Presentaban el hallazgo, en al menos dos ocasiones, de cifras de PIO superiores a $21 \mathrm{~mm} \mathrm{Hg}$, así como un estudio de PA normal. Se realizó paquimetría a todos los sujetos de este grupo. El valor medio obtenido fue de $567,7 \pm 36,9 \mu \mathrm{m}$.

- Grupo Glaucoma $(\mathrm{n}=102$ ojos). Presentaban cifras tensionales elevadas (PIO > $21 \mathrm{~mm} \mathrm{Hg}$ ) y la existencia de defectos campimétricos en la PA.

Para evitar la influencia de los datos morfométricos papilares en el análisis de los resultados, los sujetos con glaucoma no fueron seleccionados en base a la evaluación clínica papilar. Los sujetos fueron clasificados como glaucomatosos basándonos en la valoración de la PA (prueba diagnóstica de referencia).

\section{Procedimiento exploratorio}

Una vez aplicados los criterios de selección, se realizó un protocolo que incluía:

- Perimetría Automatizada Convencional (PA), realizada con el Analizador de Campo «HUMPHREY 630» (24-II, STACPAC 2). Los criterios seleccionados para definir una perimetría como alterada fueron la presencia de grupos de puntos o «clusters» con descensos significativos de sensibilidad (al menos tres puntos contiguos en el mismo hemicampo con nivel de significación $\mathrm{p}<0,05$ o un grupo de al menos dos puntos contiguos en el mismo hemicampo con nivel de significación p<0,01) (21) y/o una Desviación Estándar Patrón Corregida (CPSD) estadísticamente significativa, a niveles de $\mathrm{p}<0,02$ y/o la presencia de Glaucoma Hemifield Test (GHT) fuera de los límites de normalidad.

- Láser Confocal de Barrido. Heidelberg Retina Tomograph - HRT II.
El estudio topográfico papilar se realizó empleando un láser confocal de barrido (HRT versión II) que permite obtener una imagen topográfica de la papila mediante una serie de imágenes de cortes ópticos de diferente profundidad.

A partir de la imagen tridimensional reconstruida, el explorador delimita el contorno del disco óptico. Éste fue esteblecido siempre por el mismo oftalmólogo, especialista en glaucoma y con experiencia en planimetría papilar (JML). La delimitación se llevó a cabo sin información del grupo de inclusión del sujeto ni de la exploración. Se definió el contorno del disco óptico como la frontera entre el contenido del nervio óptico y el anillo escleral de Elschnig.

Tras ello, el programa establece un plano de referencia, 50 micras por debajo de la altura del contorno retiniano en el sector temporal entre $\operatorname{los} 350^{\circ} \mathrm{y}$ $356^{\circ}(22)$.

El HRT II incorpora un programa de análisis papilar (Heidelberg Eye Explorer version 1.3.0.0) que calcula múltiples parámetros estructurales del nervio óptico. Así, a partir del contorno papilar y del plano de referencia, el programa aporta los resultados cuantitativos morfométricos de los siguientes parámetros papilares (23):

- Área de disco, Área de excavación, Área de anillo, Cociente área de excavación/disco, Cociente área de anillo/disco, Volumen de excavación, Volumen de anillo, Profundidad media de la excavación, Profundidad máxima de la excavación, Variación de la altura del contorno, Índice de morfología de la excavación («cup shape measure»-CSM), Espesor de la capa de fibras nerviosas de la retina, Área de sección de la capa de fibras nerviosas de la retina, Cociente excavación/papila horizontal, Cociente excavación / papila vertical, Máxima elevación del contorno, Máxima depresión del contorno, Línea de modulación de contorno Temporal Superior, Línea de modulación de contorno Temporal Inferior, Variabilidad media, Altura de referencia.

Adicionalmente, el programa incorpora las siguientes funciones discriminantes:

- Función discriminante FSM: Propuesta por FS Mikelberg (24). Incluye el CSM, la Variación de la altura de contorno y el Volumen de anillo neurorretiniano, y

- Función discriminante RB: Propuesta por R. Bathija (25). Incluye el CSM, la Variación de la altura de contorno, el Espesor de la capa de fibras nerviosas de la retina y el Área de anillo neurorretiniano. 


\section{Estadística}

Se establecieron las correlaciones existentes entre dichos parámetros estructurales y los índices globales de campo visual de la PA, en cada grupo de sujetos.

Para ello, se realizó un análisis de correlación. Posteriormente al estudio de las variables cuantitativas se realizó un test de Kolmogorov-Smirnov comprobando que las muestras a estudio presentaban una distribución de sus valores ajustada a la normalidad. El coeficiente de correlación de Pearson mide la intensidad de la relación lineal entre ambas variables en caso de utilizar test parámetri- cos. Se ha utilizado el programa SPSS 11.0 (SPSS Inc, Chicago, Illinois, USA) para el tratamiento estadístico de los datos, y el programa Microsoft Word para el tratamiento de textos.

\section{RESULTADOS}

Se han incluido en el presente estudio un total de 450 ojos de 450 sujetos, 101 controles, 247 hipertensos oculares y 102 glaucomatosos. En la tabla I se recogen las características descriptivas de las poblaciones incluidas. Al comparar estas características entre grupos, existieron diferencias estadísti-

Tabla I. Características de la población

\begin{tabular}{lcccccc}
\hline & \multicolumn{2}{c}{ Grupo Control (n=101) } & \multicolumn{2}{c}{ Grupo HTO (n=247) } & \multicolumn{2}{c}{ Grupo Glaucoma (n=102) } \\
& \multicolumn{2}{c}{ Media } & D. E. & Media & D. E. & \multicolumn{2}{c}{ Media } & D. E. \\
\hline Edad & $55,53(3)$ & 12,78 & $53,7(3)$ & 12,39 & $62,9(1,2)$ & 9,70 \\
A.V. & 0,857 & 0,188 & 0,94 & 0,09 & 0,85 & 0,12 \\
PIO basal & $14,6(2,3)$ & 2,79 & $23,8(1)$ & 3,21 & $24,2(1)$ & 4,53 \\
Excavación & $0,87 / 5(2,3)$ & $0,82 / 5$ & $2,55 / 5(1,3)$ & $0,95 / 5$ & $3,46 / 5(1,2)$ & $1,12 / 5$ \\
CC-DM & $-0,56(3)$ & 1,54 & $-0,38(3)$ & 1,29 & $-6,81(1,2)$ & 6,79 \\
CC-CPSD & $1,03(3)$ & 1,06 & $1,11(3)$ & 0,90 & $4,95(1,2)$ & 3,75 \\
\hline \hline
\end{tabular}

Diferencias estadísticamente significativas: (1) respecto al grupo Control; (2) respecto al grupo Hipertenso; (3) respecto al grupo Glaucoma.

Tabla II. Coeficientes de correlación (r de Pearson) y nivel de significación entre los diferentes parámetros morfológicos papilares evaluados con el HRT II y los índices de campo visual de la perimetría automatizada convencional (DM y CPSD) en la totalidad de los sujetos muestrales

\begin{tabular}{lcccc}
\hline & & DM & CPSD & P \\
\hline cup area & Correlación de Pearson & P & Correlación de Pearson & 0,000 \\
rim area & $-0,264$ & 0,000 & 0,226 & 0,000 \\
cup/disc area ratio & $\mathbf{0 , 3 2 5}$ & 0,000 & $\mathbf{- 0 , 3 5 9}$ & 0,000 \\
rim/disc area ratio & $-0,338$ & 0,000 & 0,335 & 0,000 \\
cup volume & 0,338 & 0,000 & $-0,335$ & 0,000 \\
rim volume & $-0,177$ & 0,000 & 0,203 & 0,057 \\
mean cup depth & 0,261 & 0,000 & $-0,284$ & 0,230 \\
maximum cup depth & $-0,054$ & 0,283 & 0,097 & 0,166 \\
height variation contour & 0,077 & 0,129 & $-0,061$ & 0,000 \\
cup shape measure & 0,038 & 0,453 & $-0,070$ & 0,000 \\
mean RNFL thickness & $\mathbf{- 0 , 3 8 6}$ & 0,000 & $\mathbf{0 , 3 7 9}$ & 0,003 \\
RNFL cross sectional area & 0,338 & 0,000 & $-0,356$ & 0,000 \\
horizontal cup/disk ratio & 0,325 & 0,000 & $-0,357$ & 0,006 \\
vertical cup/disk ratio & $-0,136$ & 0,007 & 0,149 & 0,227 \\
maximum contour elevation & $-0,294$ & 0,000 & 0,282 & 0,000 \\
maximum contour depression & $-0,188$ & 0,013 & 0,139 & 0,000 \\
CLM temporal-superior & $-0,126$ & 0,000 & 0,061 & 0,025 \\
CLM temporal-inferior & 0,319 & 0,000 & $-0,303$ & 0,033 \\
average variability (SD) & 0,352 & 0,000 & $-0,402$ & 0,000 \\
reference height & $-0,162$ & 0,001 & 0,114 & 0,000 \\
FSM discriminant function value & 0,039 & 0,439 & $-0,108$ & $\mathbf{- 0 , 3 6 3}$ \\
RB discriminant function value & $\mathbf{0 , 3 6 2}$ & 0,000 & $\mathbf{- 0 , 4 4 5}$ & 0,000 \\
\hline \hline
\end{tabular}


camente significativas en los valores de PIO medias, excavación papilar, y en los valores de los índices globales perimétricos (DM y CPSD).

En el análisis realizado en la totalidad de la población muestral incluida en el estudio (normales, hipertensos oculares y glaucomas) se ha evidenciado una correlación significativa (coeficiente de correlación $\mathrm{r}$ de Pearson, $\mathrm{p}<0,01$ ) entre la mayor parte de los parámetros papilares, excepto el área de disco, la profundidad media y máxima de la excavación, y la variación de la altura de contorno, y los índices de campo visual (DM y CPSD) de la PA (tabla II).

De los diferentes parámetros evaluados, el área de anillo, el índice de morfología de la excavación y las funciones discriminantes FSM y RB han mos-

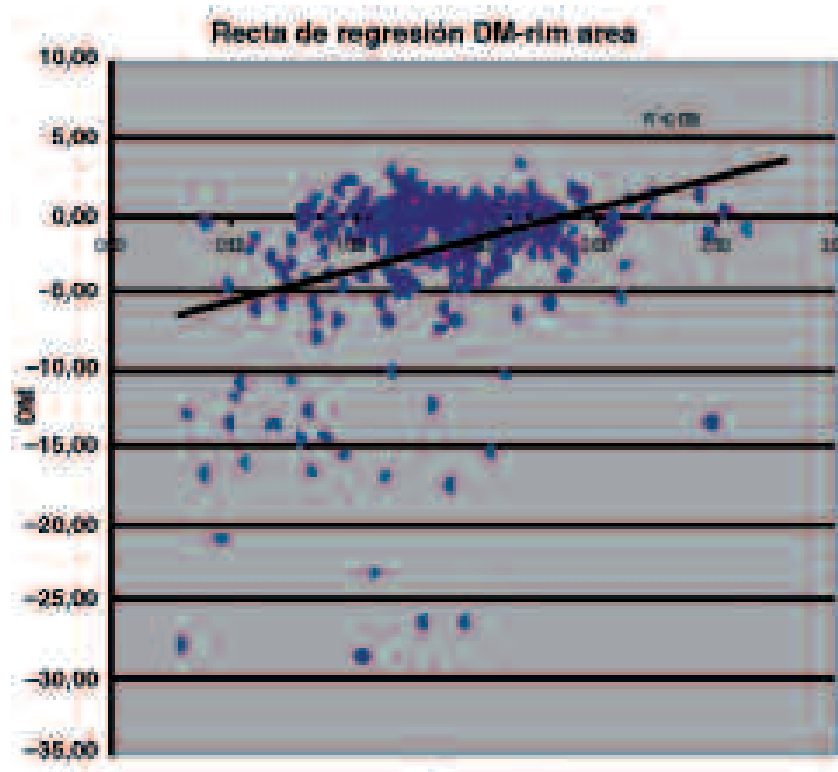

rim area

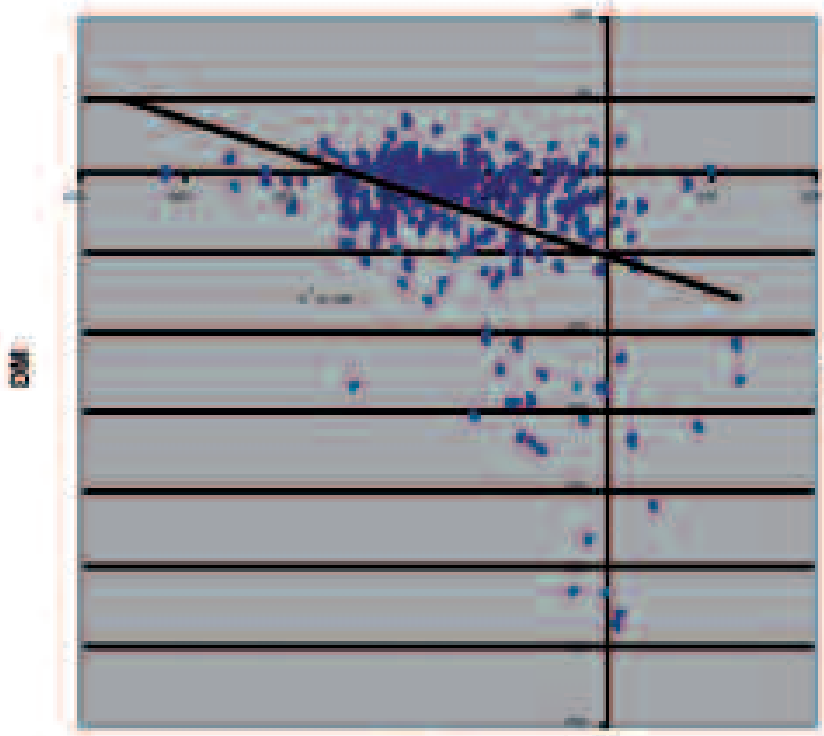

cam

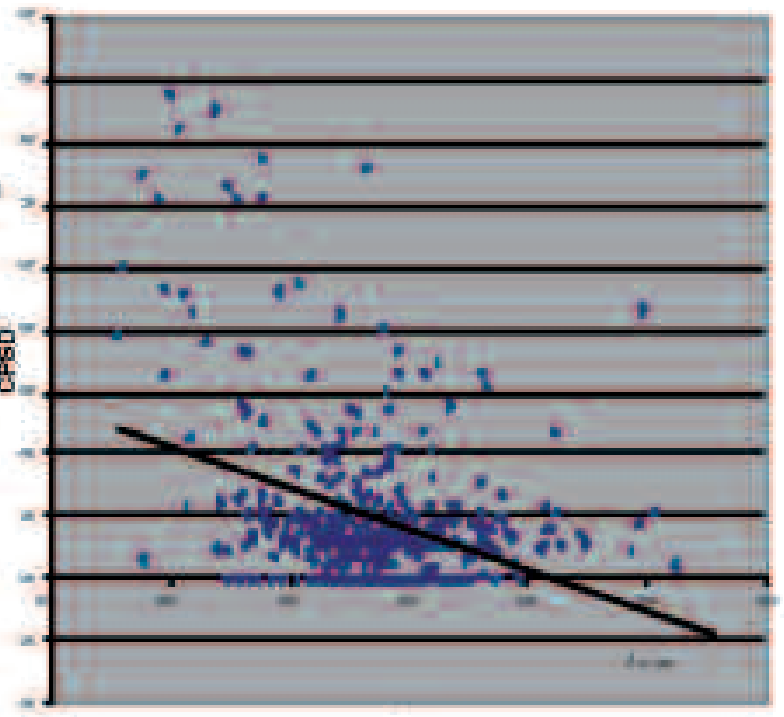

rim ares

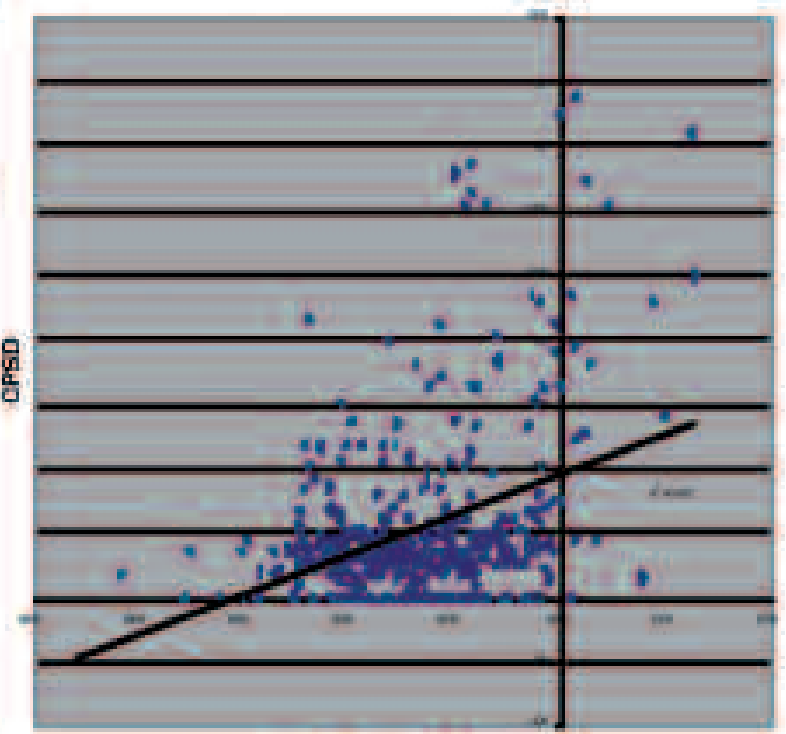

eup shape messure

Fig. 1: Recta de regresión resultante de la correlación entre el área de anillo, el índice de morfología de la excavación (cup shape measure), la función discriminante FSM y la función discriminante RB y los índices de campo visual de la perimetría automatizada convencional (DM y CPSD) en la totalidad de los sujetos muestrales. 


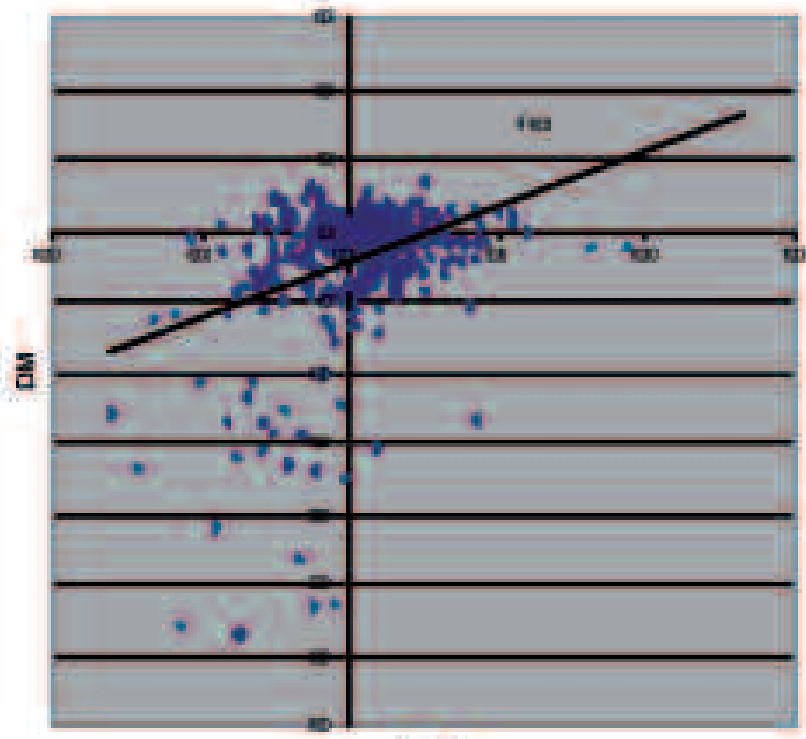

FBS

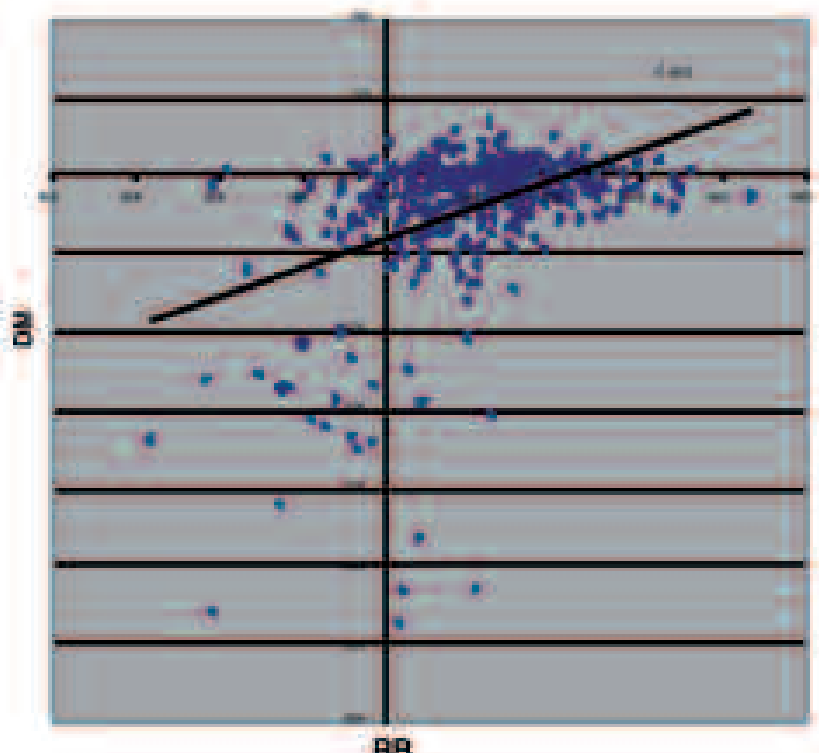

AB
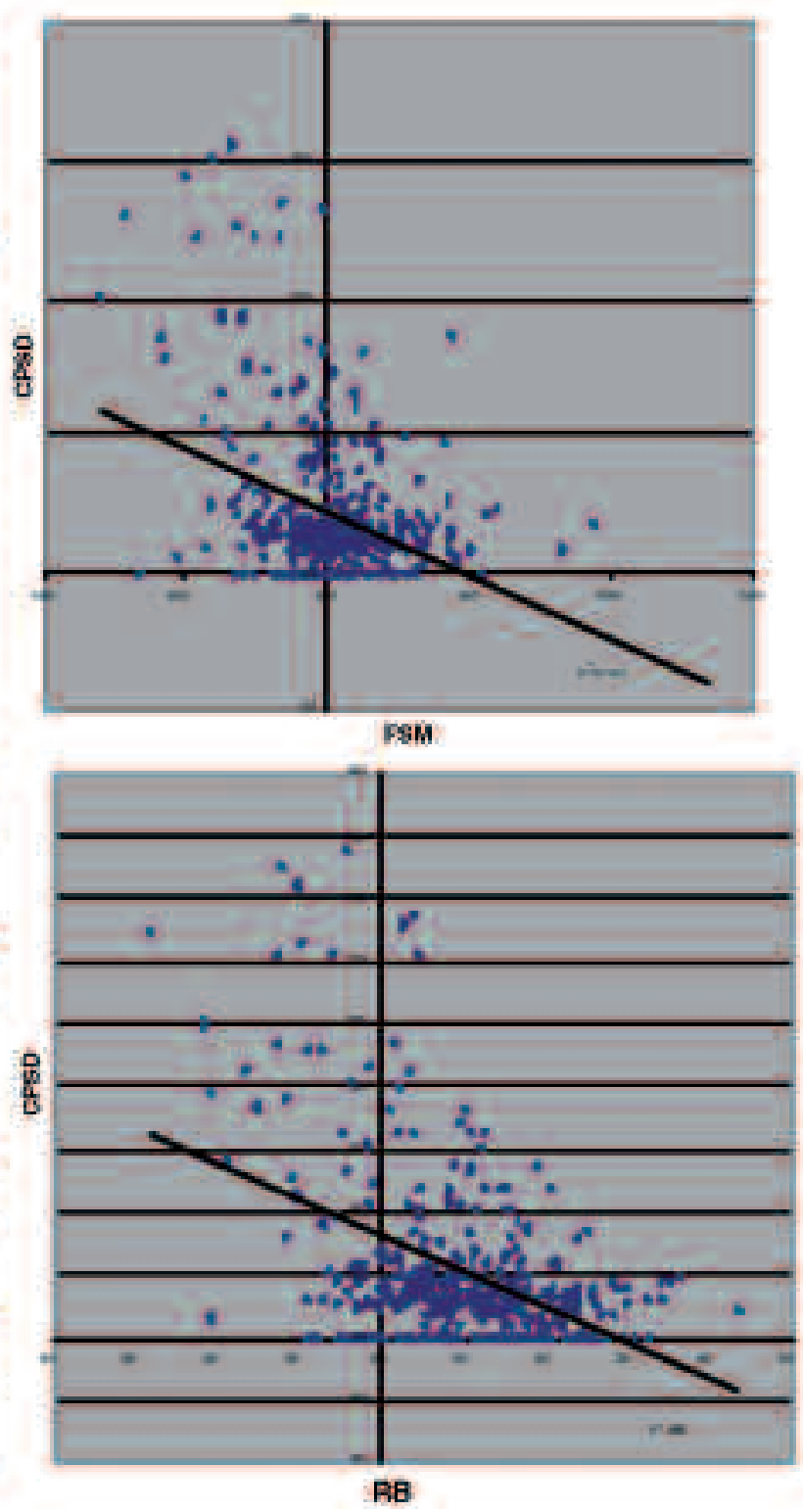

Fig. 1 (continuación).

trado las correlaciones más elevadas con los índices de campo visual (DM y CPSD) (fig. 1).

Analizando la correlación existente entre los parámetros papilares y los índices de campo visual en el grupo de sujetos normales, no se ha evidenciado una correlación significativa entre ninguno de los parámetros y los índices de la PA.

Por el contrario, en relación a la población con glaucoma se ha evidenciado una correlación significativa (coeficiente de correlación $\mathrm{r}$ de Pearson, $\mathrm{p}<$ 0,05) entre los índices de campo visual (DM y
CPSD) de la PA y el área de excavación y de anillo, el cociente de área excavación/disco, el volumen de anillo, el CSM, el espesor medio de la CFNR, el área de sección de la CFNR, el cociente vertical área excavación/disco, la modulación de la línea de contorno temporal superior e inferior y las funciones discriminantes FSM y RB (tabla III). De entre todos estos parámetros el CSM, el cociente excavación/disco vertical y las funciones discriminante FSM y RB han mostrado las correlaciones más elevadas con los índices de campo visual (DM y CPSD) (fig. 2). 
Dentro del grupo de sujetos hipertensos oculares sólo se ha evidenciado una correlación cerca de ser significativa entre el cociente del área excavación/disco y el cociente del área anillo/disco con la CPSD de los índices de campo visual de la PA (tabla IV).

\section{DISCUSIÓN}

A pesar de la diferente naturaleza de los parámetros estructurales obtenidos con el HRT II y los datos funcionales de los diversos métodos de exploración del campo visual, intuitivamente cabe esperar una correlación entre los resultados de ambos tipos de pruebas, que dependerá del tipo de muestra seleccionada.

En nuestro estudio, realizamos un análisis en los distintos grupos muestrales, de la correlación existente entre los parámetros estructurales obtenidos mediante HRT II, y los índices de la PA (DM y CPSD).

En relación a los resultados, el hecho de que únicamente obtengamos correlaciones significativas cuando incluimos en la muestra sujetos con perimetría alterada (glaucomas) se explica probablemente, por la amplitud del rango de valores numéricos que en este grupo se obtienen en los índices DM y CPSD, cosa que no ocurre en ninguno de los demás grupos por obtener resultados normales y por tanto de valores muy acotados, con los que no se puede llegar a establecer una correlación a partir de los valores numéricos de los parámetros del HRT, los cuales sí varían ampliamente.

Teniendo en cuenta que el modelo de relación entre anatomía y función no tiene porqué ser siempre lineal en todos los tramos de la distribución y que dicha distribución depende de la muestra que se emplea, se podrían desarrollar estudios que incluyan ajustes cuadráticos y cúbicos que posiblemente mostrarían una mejor correlación entre los parámetros empleados.

Al comparar nuestros resultados con los de otros autores que también han estudiado estos aspectos, podemos decir que Eid et al, Mistlberger et al, Tsai et al, Iester et al y Brigatti et al (26-30) también obtienen correlaciones significativas en distintos parámetros, pero en todos sus trabajos se encuentran o en el grupo de sujetos con glaucoma, o en la muestra poblacional completa. Ninguno de ellos encuentra correlación en los sujetos normales o en los hipertensos oculares. Existe un trabajo de Iester

Tabla III. Coeficientes de correlación ( $r$ de Pearson) y nivel de significación entre los diferentes parámetros morfológicos papilares evaluados con el HRT II y los índices de campo visual de la perimetría automatizada convencional (DM y CPSD) en los sujetos glaucomatosos

\begin{tabular}{|c|c|c|c|c|}
\hline & \multicolumn{2}{|c|}{ DM } & \multicolumn{2}{|c|}{ CPSD } \\
\hline & Correlación de Pearson & $\mathrm{P}$ & Correlación de Pearson & $\mathrm{P}$ \\
\hline cup area & $-0,281$ & 0,004 & 0,212 & 0,034 \\
\hline rim area & 0,286 & 0,004 & $-0,341$ & 0,000 \\
\hline cup/disc area ratio & $-0,338$ & 0,001 & 0,336 & 0,001 \\
\hline $\mathrm{rim} / \mathrm{disc}$ area ratio & 0,338 & 0,001 & $-0,336$ & 0,001 \\
\hline cup volume & $-0,122$ & 0,222 & 0,183 & 0,067 \\
\hline rim volume & 0,268 & 0,006 & $-0,276$ & 0,005 \\
\hline mean cup depth & $-0,038$ & 0,704 & 0,124 & 0,216 \\
\hline maximum cup depth & 0,082 & 0,414 & $-0,061$ & 0,544 \\
\hline height variation contour & $-0,042$ & 0,676 & 0,028 & 0,780 \\
\hline cup shape measure & $-0,434$ & 0,000 & 0,450 & 0,000 \\
\hline mean RNFL thickness & 0,318 & 0,001 & $-0,338$ & 0,001 \\
\hline RNFL cross sectional area & 0,295 & 0,003 & $-0,337$ & 0,001 \\
\hline horizontal cup/disk ratio & $-0,156$ & 0,118 & 0,184 & 0,065 \\
\hline vertical cup/disk ratio & $-0,362$ & 0,000 & 0,332 & 0,001 \\
\hline maximum contour elevation & $-0,206$ & 0,038 & 0,120 & 0,233 \\
\hline maximum contour depression & $-0,242$ & 0,014 & 0,145 & 0,149 \\
\hline CLM temporal-superior & 0,319 & 0,001 & $-0,268$ & 0,007 \\
\hline CLM temporal-inferior & 0,295 & 0,003 & $-0,405$ & 0,000 \\
\hline average variability (SD) & $-0,130$ & 0,194 & 0,088 & 0,379 \\
\hline reference height & $-0,030$ & 0,763 & $-0,102$ & 0,311 \\
\hline FSM discriminant function value & 0,378 & 0,000 & $-0,390$ & 0,000 \\
\hline RB discriminant function value & $\mathbf{0 , 3 8 4}$ & 0,000 & $-0,429$ & 0,000 \\
\hline
\end{tabular}




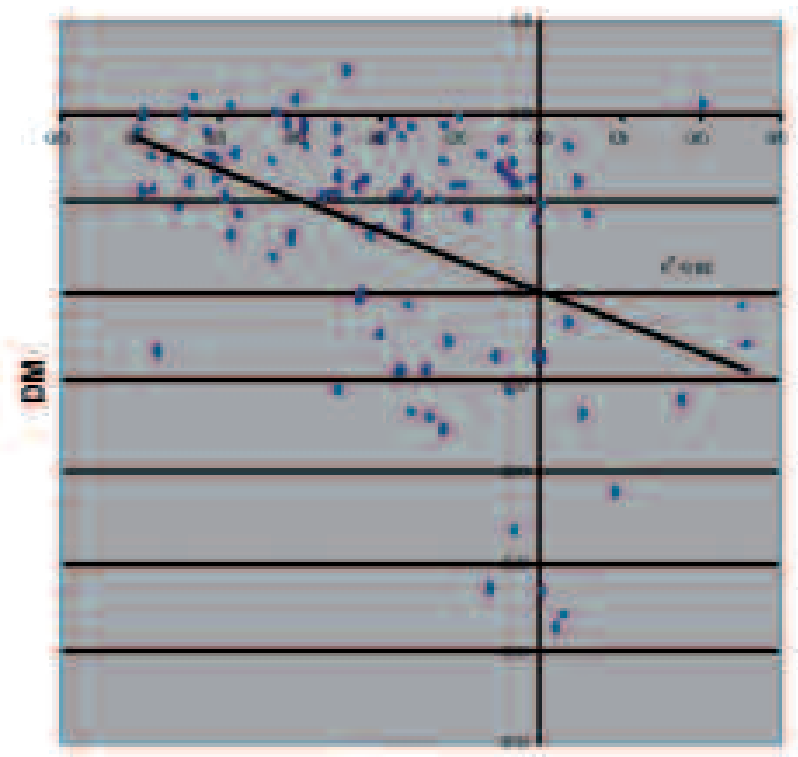

csa

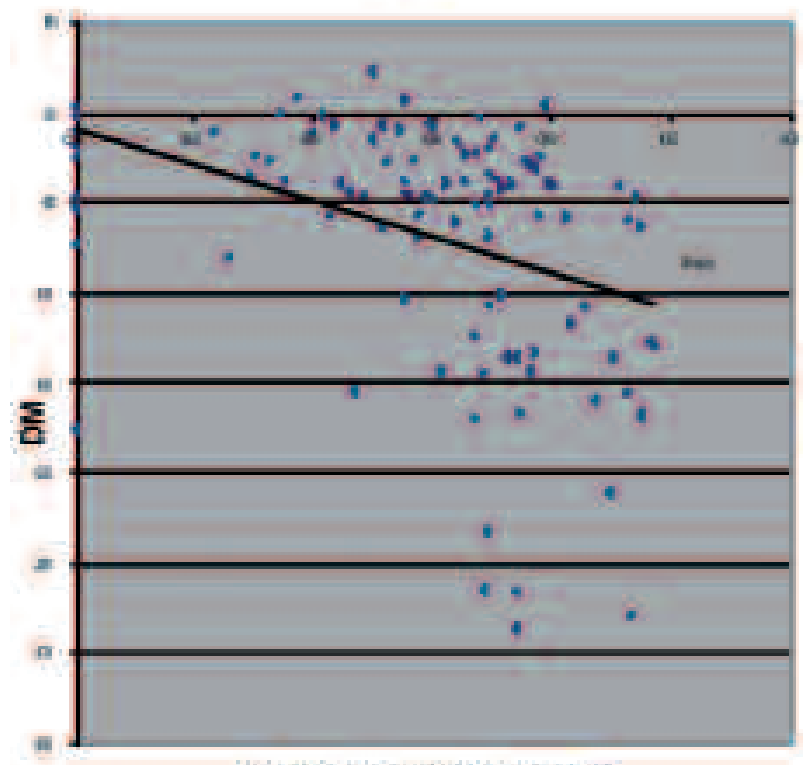

VERTICAL CUPDISK RATIO
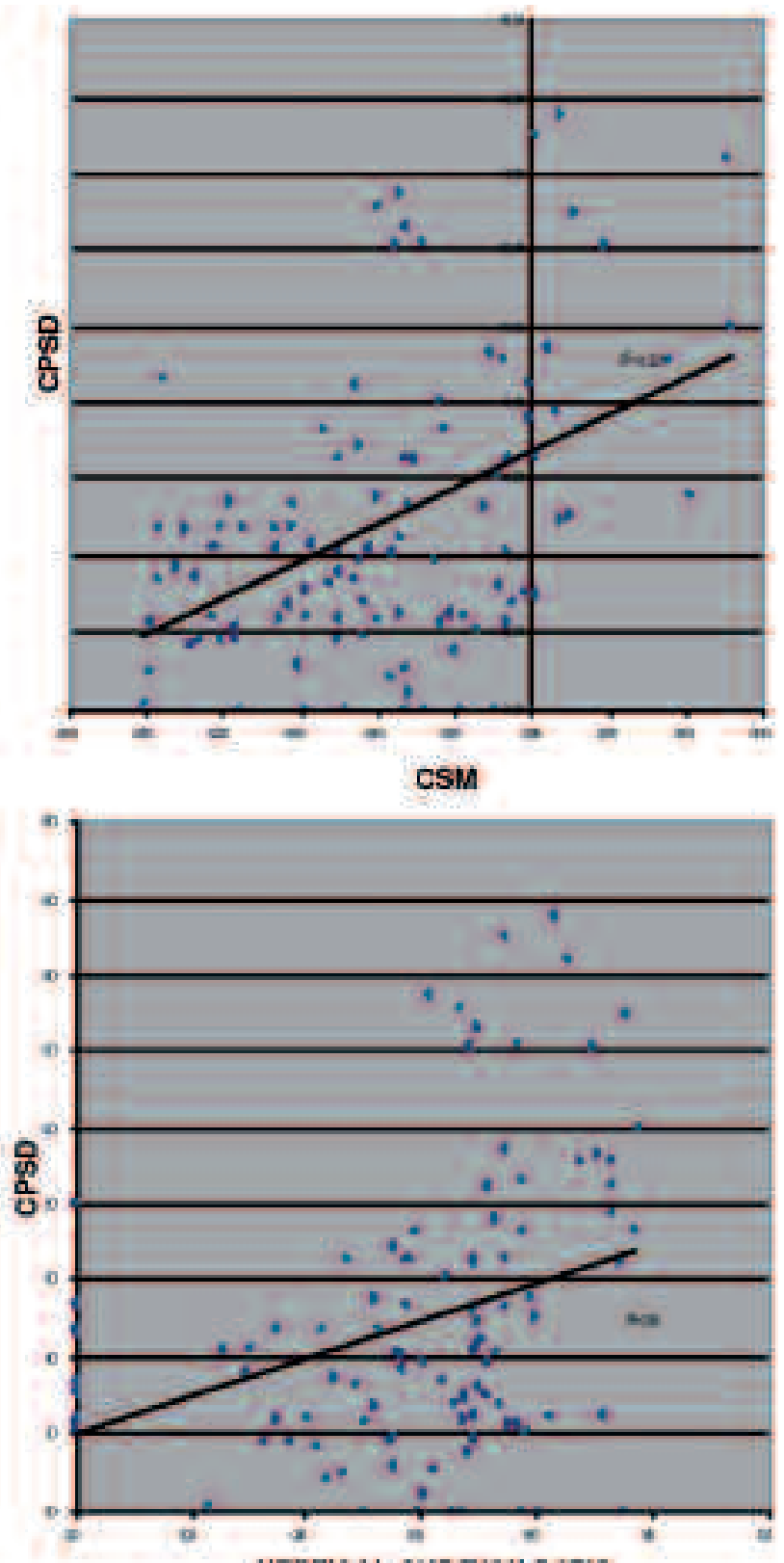

VEATICAL CUPDISK AATIO

Fig. 2: Recta de regresión resultante de la correlación entre el cociente excavación/disco vertical, el índice de morfología de la excavación (cup shape measure), la función discriminante FSM y la función discriminante RB y los índices de campo visual.

et al (31) en el que sí hay correlación en dos de los parámetros estructurales obtenidos en el grupo de hipertensos oculares (con campo visual normal), y los índices de la perimetría. Éstos son el área de anillo y el volumen de anillo.

Si nos centramos en los parámetros en que cada uno de los autores obtiene mayor correlación dire- mos que, Brigatti y col. (30) observaron correlaciones estadísticamente significativas del CSM tanto con la DM como con la CPSD. Según Iester y Mikelberg (31), el área de anillo neurorretiniano es el parámetro con mejor correlación con la CPSD y en menor medida con la DM. Mistlberger (27) encontró correlación entre el espesor de la CFNR y la DM. 

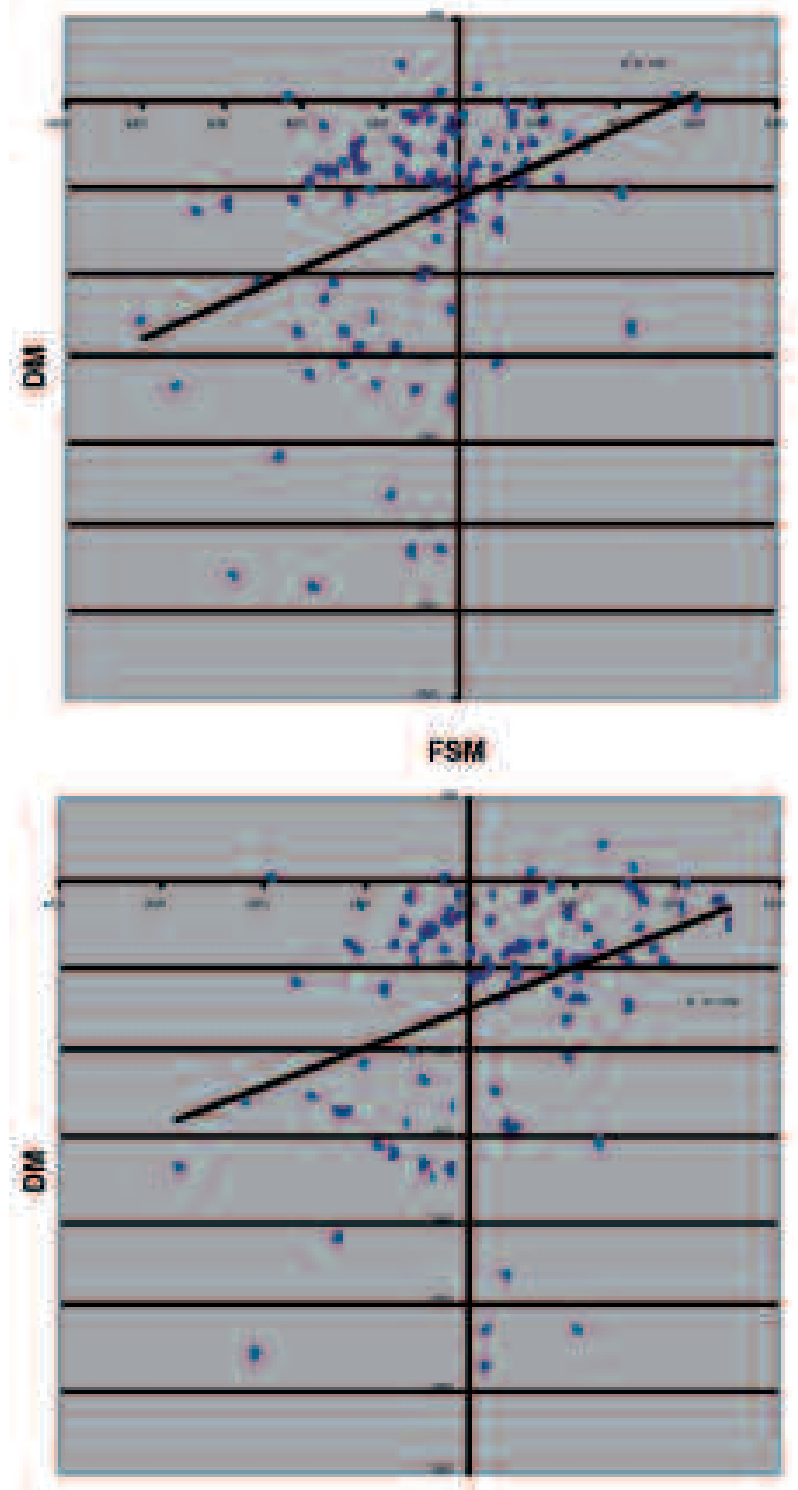

$\mathrm{AB}$

Fig. 2 (continuación).

Uchida et al (32) hablan fundamentalmente de la buena correlación del CSM con la DM y la CPSD.

Los topógrafos papilares pueden obtener resultados sectoriales de las características del nervio óptico. Esta posibilidad ha permitido analizar por sectores las características topográficas papilares y su correlación con los índices de campo visual (29). Los resultados de este estudio ofrecen correlaciones más fuertes entre los parámetros topográficos papilares de los sectores superiores e inferiores y los índices de sus correspondientes regiones de campo
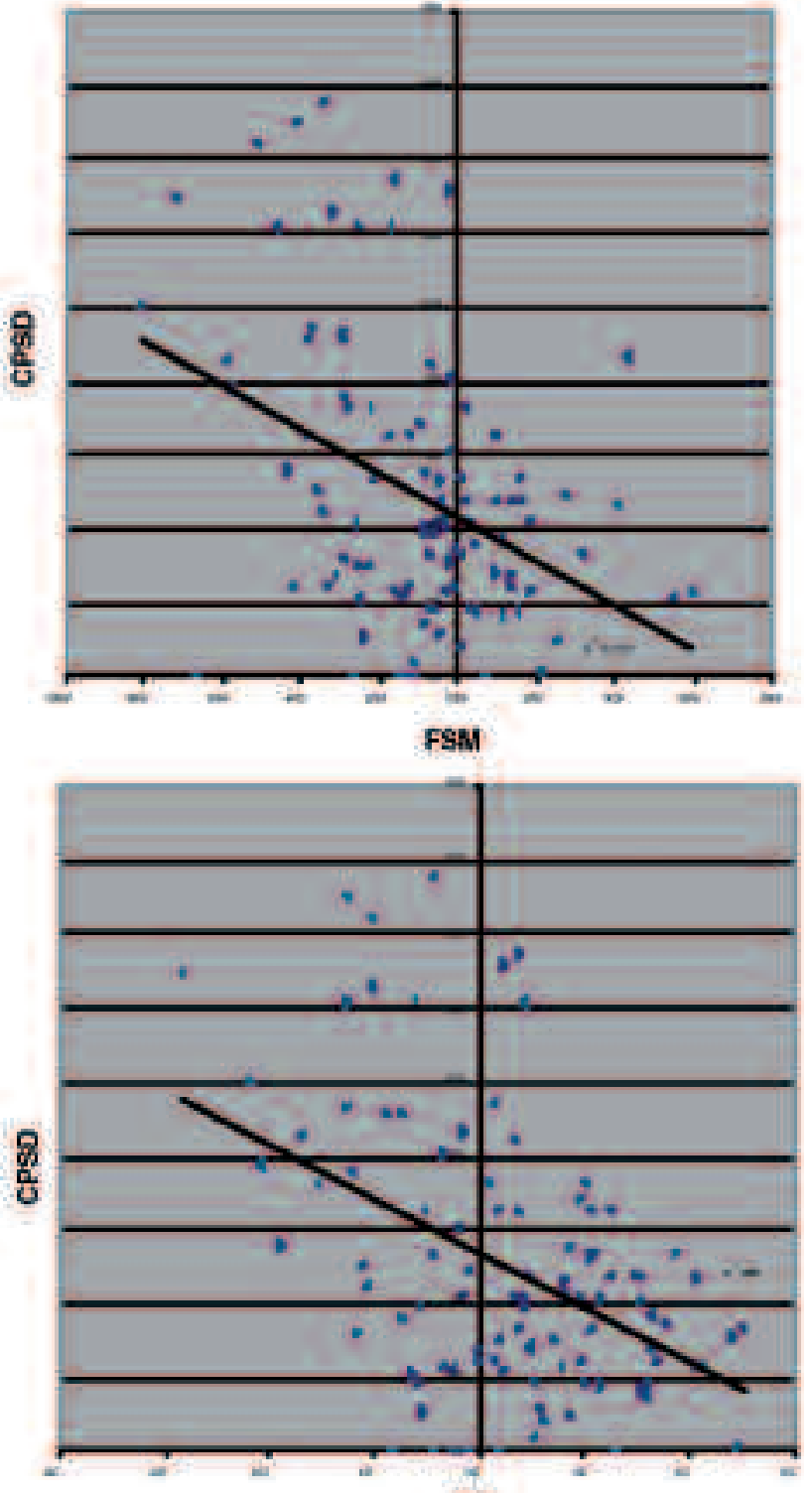

RB

visual, mientras que los sectores nasales y temporales obtuvieron unas correlaciones más débiles. Las correlaciones más estrechas se obtuvieron entre los parámetros topográficos del sector inferior con la DM del hemicampo superior. Los parámetros sectoriales que mostraron asociaciones más fuertes fueron el CSM y el área de la excavación. Otros autores como Antón et al (33) han analizado la relación topográfica entre localizaciones específicas del campo visual glaucomatoso y el daño del nervio óptico. En su estudio concluyen que el cociente de 
Tabla IV. Coeficientes de correlación (r de Pearson) y nivel de significación entre los diferentes parámetros morfológicos papilares evaluados con el HRT II y los índices de campo visual de la perimetría automatizada convencional (DM y CPSD) en los sujetos hipertensos oculares

\begin{tabular}{lcccc}
\hline & & DM & & CPSD \\
& Correlación de Pearson & P & Correlación de Pearson & P \\
\hline cup area & 0,037 & 0,677 & $-0,160$ & 0,074 \\
rim area & $-0,129$ & 0,151 & 0,069 & 0,443 \\
cup/disc area ratio & 0,077 & 0,390 & $\mathbf{- 0 , 1 7 2}$ & 0,054 \\
rim/disc area ratio & $-0,077$ & 0,390 & $\mathbf{0 , 1 7 2}$ & 0,054 \\
cup volume & 0,054 & 0,545 & $-0,145$ & 0,106 \\
rim volume & $-0,157$ & 0,079 & 0,004 & 0,965 \\
mean cup depth & 0,097 & 0,282 & $-0,162$ & 0,069 \\
maximum cup depth & 0,106 & 0,236 & $-0,161$ & 0,071 \\
height variation contour & $-0,107$ & 0,235 & $-0,109$ & 0,223 \\
cup shape measure & $-0,010$ & 0,915 & $-0,052$ & 0,561 \\
mean RNFL thickness & $-0,063$ & 0,481 & $-0,072$ & 0,423 \\
RNFL cross sectional area & $-0,089$ & 0,319 & $-0,076$ & 0,395 \\
horizontal cup/disk ratio & 0,111 & 0,217 & $-0,112$ & 0,211 \\
vertical cup/disk ratio & 0,087 & 0,330 & $-0,135$ & 0,131 \\
maximum contour elevation & $-0,071$ & 0,431 & $-0,121$ & 0,178 \\
maximum contour depression & $-0,115$ & 0,198 & $-0,152$ & 0,088 \\
CLM temporal-superior & $-0,019$ & 0,835 & $-0,024$ & 0,792 \\
CLM temporal-inferior & $-0,033$ & 0,713 & $-0,061$ & 0,495 \\
average variability (SD) & $-0,140$ & 0,117 & $-0,115$ & 0,201 \\
reference height & $-0,095$ & 0,288 & $-0,133$ & 0,137 \\
FSM discriminant function value & $-0,109$ & 0,226 & 0,103 & 0,251 \\
RB discriminant function value & 0,006 & 0,941 & $-0,051$ & 0,573 \\
\hline \hline
\end{tabular}

área ANR/D es un parámetro útil para objetivar daño focal del anillo, y parece compensar la variabilidad interindividual en la configuración de la cabeza del nervio óptico.

Este estudio establece la existencia de correlación significativa (aunque no muy elevada) entre parámetros estructurales y funcionales en el grupo de sujetos glaucomatosos de nuestra amplia muestra poblacional. Esto podría apoyar la teoría de que en sujetos con glaucoma el daño funcional local ya está establecido y hay una mayor coherencia con el daño estructural de su nervio óptico y CFNR, algo que no ocurre en los sujetos hipertensos o normales, con campos visuales normales. En el grupo de sujetos hipertensos oculares sí se observan alteraciones en algunos parámetros estructurales obtenidos por el HRT II, lo cual hace pensar en un defecto anatómico más precoz que el daño funcional en este grupo de sujetos y esto explica la mala correlación entre los índices de ambas pruebas.

En general, las correlaciones existentes permiten mejorar el conocimiento del daño glaucomatoso y facilitan la toma de decisiones desde una posición coherente con los resultados de todas las pruebas funcionales y estructurales de las que disponemos en la actualidad.

\section{BIBLIOGRAFÍA}

1. Jonas JB, Fernandez MC, Sturmer J. Pattern of glaucomatous neuroretinal rim loss. Ophthalmology 1993; 100: 63-68.

2. Polo V, Abecia E, Pablo LE, Pinilla I, Larrosa JM, Honrubia FM. Short-wavelength automated perimetry and retinal nerve fiber layer evaluation in suspected cases of glaucoma. Arch Ophthalmol 1998; 116: 1295-1298.

3. Sommer A, Katz J, Quigley HA, Miller NR, Robin AL, Richter $R C$, et al. Clinically detectable nerve fiber atrophy predeces the onset of glaucomatous field loss. Arch Ophthalmol 1991; 109: 77-83.

4. Heijl A, Lindgren G, Olsson J, Asman P. Visual field interpretation with empiric probability maps. Arch Ophthalmol 1989; 107: 204-208.

5. Polo V, Larrosa JM, Pinilla I, Perez S, Gonzalvo F, Honrubia FM. Predictive value of short-wavelength automated perimetry: a 3-year follow-up study. Ophthalmology 2002; 109: 761-765.

6. Balazsi AG, Drance SM, Schulzer M, Douglas GR. Neuroretinal rim area in suspected glaucoma and early chronic open-angle glaucoma. Correlation with parameters of visual function. Arch Ophthalmol 1984; 102: 1011-1014.

7. Airaksinen PJ, Drance SM, Schulzer M. Neuroretinal rim area in early glaucoma. Am J Ophthalmol 1985; 99: 1-4.

8. Tuulonen A, Airaksinen PJ. Initial glaucomatous optic disk and retinal nerve fiber layer abnormalities and their progression. Am J Ophthalmol 1991; 111: 485-490.

9. Airaksinen PJ, Tuulonen A, Alanko HI. Rate and pattern of neuroretinal rim area decrease in ocular hypertension and glaucoma. Arch Ophthalmol 1992; 110: 206-210. 
10. Jonas JB, Konigsrereuther KA. Optic disc appearance in ocular hypertensive eyes. Am J Ophthalmol 1994; 117: 732-740.

11. Heilj A, Bengtsson B. The effect of perimetric experience in patients with glaucoma. Arch Ophthalmol 1996; 114: 19-22.

12. Quigley HA, Addicks EM, Green WR. Optic nerve damage in human glaucoma. III. Quantitative correlation of nerve fiber loss and visual field defect in glaucoma, ischemic neuropathy, papilledema, and toxic neuropathy. Arch Ophthalmol 1982; 100: 135-146.

13. Sommer A, Katz J, Quigley HA, Miller NR, Robin A, Richter $R C$, et al. Clinically detectable nerve fiber atrophy precedes the onset of glaucomatous field loss. Arch Ophthalmol 1991; 109: 77-83.

14. Garway-Heath DF, Poinoosawmy D, Wollstein G, Viswanathan A, Kamal D, Fontana L, et al. Inter- and intraobserver variation in the analysis of optic disc images: comparison of the Heidelberg retina tomograph and computer assisted planimetry. Br J Ophthalmol 1999; 83: 664-669.

15. Rohrschneider K, Burk RO, Kruse FE, Volcker HE. Reproducibility of the optic nerve head topography with a new laser tomographic scanning device. Ophthalmology 1994; 101: 1044-1049.

16. Janknecht P, Funk J. Optic nerve head analyser and Heidelberg retina tomograph: accuracy and reproducibility of topographic measurements in a model eye and in volunteers. Br J Ophthalmol 1994; 78: 760-768.

17. Weinreb RN, Lusky M, Bartsch DU, Morsman D. Effect of repetitive imaging on topographic measurements of the optic nerve head. Arch Ophthalmol 1993; 111: 636-638.

18. Hatch WV, Flanagan JG, Williams-Lyn DE, Buys YM, Farra T, Trope G E. Interobserver agreement of Heidelberg retina tomograph parameters. J Glaucoma 1999; 8: 232-237.

19. Nakla M, Nduaguba C, Rozier M, Joudeh M, Hoffman D, Caprioli J. Comparison of imaging techniques to detect glaucomatous optic nerve damage. Invest Ophthalmol Vis Sci 1999; 40: S397.

20. Zangwill LM, Bowd C, Berry CC, Williams J, Blumenthal EZ, Sanchez-Galeana CA, et al. Discriminating between normal and glaucomatous eyes using the Heidelberg Retina Tomograph, GDx Nerve Fiber Analyzer, and Optical Coherence Tomograph. Arch Ophthalmol 2001; 119: 985993.

21. Caprioli J. Automated perimetry in glaucoma. Am J Ophthalmol 1991; 111: 235-239.
22. Vihanninjoki K, Burk RO, Teesalu P, Tuulonen A, Airaksinen PJ. Optic disc biomorphometry with the Heidelberg Retina Tomograph at different reference levels. Acta Ophthalmol Scand 2002; 80: 47-53.

23. Saruhan A, Orgul S, Kocak I, Prunte C, Flammer J. Descriptive information of topographic parameters computed at the optic nerve head with the Heidelberg retina tomograph. J Glaucoma 1998; 7: 420-429.

24. Mikelberg FS, Parfitt CM, Swindale NV, Graham SL, Drance SM, Gosine R. Ability of the Heidelberg retina tomograph to detect early glaucomatous visual field loss. J Glaucoma 1995; 4: 242-247.

25. Bathija R, Zangwill L, Berry CC, Sample PA, Weinreb RN. Detection of early glaucomatous structural damage with confocal scanning laser tomography. J Glaucoma 1998; 7: 121-127.

26. Eid TM, Spaeth GL, Katz LJ, Azuara-Blanco A, Agusburger J, Nicholl J. Quantitative estimation of retinal nerve fiber layer height in glaucoma and the relationship with optic nerve head topography and visual field. J Glaucoma 1997; 6: 221-230.

27. Mitslberger A, Liebmann JM, Greenfield DS, Pons ME, Hoh ST, Ishikawa H, et al. Heidelberg retina tomography and optical coherence tomography in normal, ocularhypertensive and glaucomatous eyes. Ophthalmology 1999; 106: 2027-2032.

28. Tsai CS, Zangwill L, Sample PA, Garden V, Bartsch DU, Weinreb RN. Correlation of peripapillary retinal height and visual field in glaucoma and normal subjects. J Glaucoma 1995; 4: 110-116.

29. Iester M, Swindale NV, Mikelberg FS. Sector-based analysis of optic nerve head shape parameters and visual field indices in healthy and glaucomatous eyes. J Glaucoma 1997; 6: 371-376.

30. Brigatti L, Caprioli J. Correlation of visual field with scanning confocal laser optic disc measurements in glaucoma. Arch Ophthalmol 1995; 113: 1191-1194.

31. Iester M, Mikelberg FS, Courtright P, Drance SM. Correlation between the visual field indices and Heidelberg retina tomograph parameters. J Glaucoma 1997; 6: 78-82.

32. Uchida H, Brigatti L, Caprioli J. Detection of structural damage from glaucoma with confocal laser image analysis. Invest Ophthalmol Vis Sci 1996; 37: 2393-2401.

33. Anton A, Yamagishi N, Zangwill L, Sample PA, Weinreb RN. Mapping structural to functional damage in glaucoma with standard automated perimetry and confocal scanning laser ophthalmoscopy. Am J Ophthalmol 1998; 125: 436-446. 